> La santé bucco-dentaire participe au maintien d'un bon état général de l'organisme. Certaines populations, dont les personnes détenues, ont des besoins importants mais, simultanément, un accès limité aux soins dentaires. Télédent est un outil complémentaire de téléexpertise buccodentaire qui facilite le dépistage et la prévention des troubles oraux. À partir d'une caméra intra-buccale qui enregistre des images qui sont analysées par l'équipe du service de médecine bucco-dentaire de l'Hôpital Henri Mondor, l'Unité sanitaire du centre pénitentiaire de Fresnes dispose d'une expertise dentaire détaillée. Télédent permet ainsi une coopération entre les services qui améliore le dépistage et la prise en charge de soins bucco-dentaires, tout en diminuant les coûts et en favorisant la sécurité des patients détenus. <

Télédent, l'activité de téléexpertise bucco-dentaire, a été mise en place à l'hôpital Henri Mondor en septembre 2018. Elle émane d'une réflexion menée depuis 2016 autour d'un enjeu majeur de santé publique : comment faciliter l'accès aux soins dentaires aux personnes détenues.

Les personnes détenues des Maisons d'arrêt, qui doivent se voir proposer un examen médical et bucco-dentaire dès leur arrivée en détention, représentent une population pleinement concernée par cet enjeu de santé publique [1]. Ces personnes, dont l'état bucco-dentaire est souvent très altéré, ont des besoins élevés en termes de prise en charge. II s'agit le plus souvent d'une population masculine jeune, avec un faible niveau socio-économique. Elles présentent fréquemment des comportements à risque, notamment une consommation de tabac, de cannabis, de cocaïne et la prise de psychotropes. Leur hygiène bucco-dentaire est souvent déficiente et le suivi dentaire insuffisant. Selon une étude effectuée en 2008 par la Direction générale de la santé (DGS) [2], l'examen bucco-dentaire d'entrée n'est effectué que dans $52 \%$ des établissements pénitentiaires.

\section{Télédent, \\ une expérience de téléexpertise bucco-dentaire en milieu pénitentiaire}

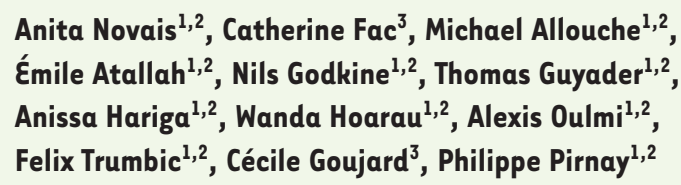

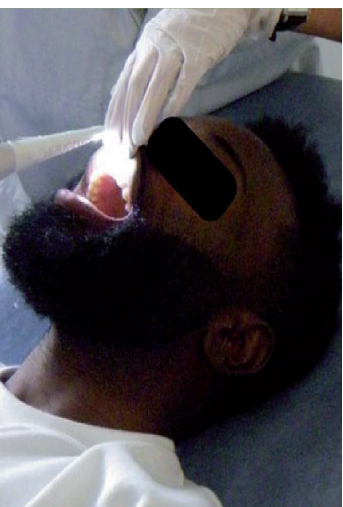

${ }^{1}$ Hôpital Henri-Mondor - Service de médecine bucco-dentaire Groupe Henri Mondor AP-HP, Île de France, Créteil, France. ${ }^{2}$ Faculté de chirurgie dentaire de I'Université Paris Descartes, 1 rue Maurice Arnoux, 92120 Montrouge, France. ${ }^{3}$ Unité sanitaire du centre pénitentiaire de Fresnes (94) CHU Bicêtre AP-HP, Île-de-France, France. anitarsnovais@gmail.com

Les soins bucco-dentaires en milieu carcéral se heurtent à de nombreuses difficultés, mais des améliorations sont possibles [2, 3]. La difficulté de recrutement de chirurgiens-dentistes, due à une rémunération peu attractive en regard d'une activité libérale, la lourdeur administrative, ainsi que les impératifs du milieu qui impliquent des délais pour les rendez-vous, pèsent sur les établissements pénitentiaires.

Dans le centre pénitentiaire de Fresnes, l'équipe de chirurgiens-dentistes est constituée de deux équivalents temps plein répartis entre plusieurs praticiens. Ce modèle de prise en charge ne permet pas de répondre aux besoins de dépistages et de soins tant le nombre de détenus est important. Télédent se propose donc de réaliser les actes de dépistage et de conseiller et optimiser la prise en charge des patients selon le degré d'urgence de leur état dentaire. En permettant aux chirurgiens-dentistes du centre pénitentiaire de soigner les patients après un dépistage réalisé par téléexpertise, Télédent optimise le parcours de prise en charge des patients et rationalise la prise de rendez-vous.

Concrètement, le praticien en charge de l'examen des personnes détenues entrantes propose ce dépistage. L'infirmière chargée du projet, à l'aide d'un dispositif et d'un protocole simple, enregistre des images de la cavité buccale du patient qui sont ensuite transmises au service de médecine bucco-dentaire de l'Hôpital Henri Mondor au sein duquel une équipe dédiée de chirurgiens-dentistes les analyse. 


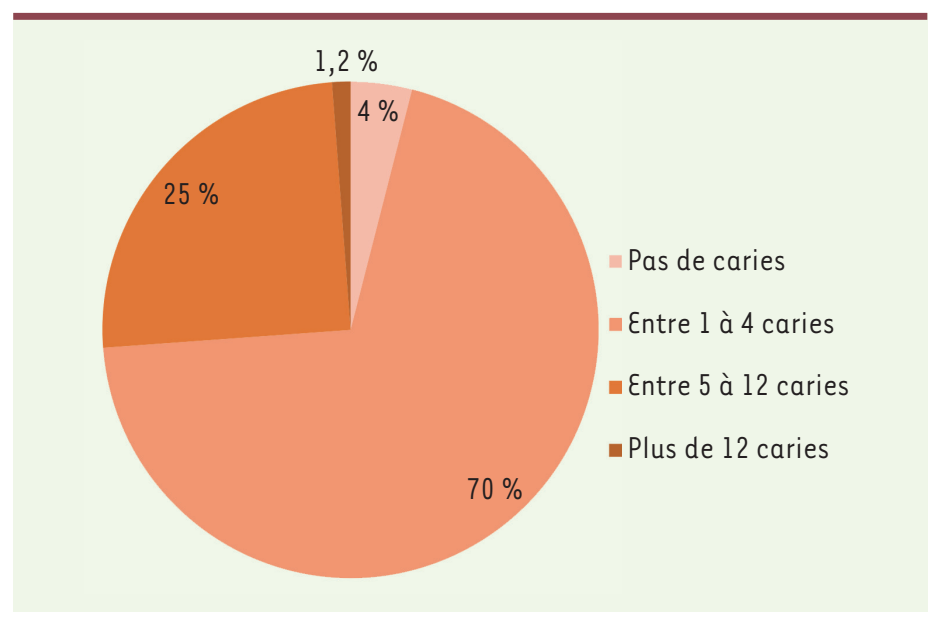

Figure 1. État bucco-dentaire des détenus.

Télédent nécessite plusieurs préalables. Le patient doit être informé et consentir à cet acte de télémédecine. Les équipes partenaires doivent être rigoureuses et formées à toutes les étapes du protocole. Et, afin que l'ensemble des acteurs opèrent en synergie, les images doivent être précises, les délais de réponse courts, le dispositif technique doit répondre aux exigences de sécurité des données du patient et la communication entre les équipes doit être fluide.

\section{Pourquoi Télédent?}

Ce service présente des caractéristiques qui lui procurent plusieurs avantages.

\section{Accès facilité à la prévention et aux soins}

Le développement de la téléexpertise bucco-dentaire permet aux résidents des établissements requérants d'accéder au dépistage et à la prévention. Télédent facilite donc la prévention et les soins dentaires. Dans des structures où le flux humain n'est parfois pas compatible avec l'analyse et le dépistage systématiques des problèmes de santé dentaire, comme le centre pénitentiaire de Fresnes, la téléexpertise offre l'opportunité à tous les entrants de profiter d'un suivi buccodentaire. Dans une étude réalisée par Fac et al. en $2016^{1}$, il a été constaté que $96 \%$ de la population à la Maison d'arrêt de Fresnes présentait des caries, dont $26,2 \%$ de patients avec plus de 5 caries en bouche (Figure 1).

\section{Diminution des déplacements et des coûts}

Un autre avantage de la téléexpertise consiste à diminuer le nombre et le coût des déplacements des accompagnants. Selon un rapport établi par le Ministère de l'intérieur, le coût total en personnel (gardes et escortes de détenus hospitalisés) était estimé en 2006 à 41 millions d'euros (Tableau 1) [4]. La téléexpertise, comme son nom l'indique, qui permet la mise en place d'une expertise à distance, offre donc

${ }^{1}$ Fac C. Partenariat entre une unité d'enseignement en odontologie et une unité sanitaire. $14^{e}$ Congrès National des UCSA, Toulouse, 28-29 mars 2019.

\begin{tabular}{lc}
\hline & $\begin{array}{c}\text { Coût total en } 2006 \\
(M €)\end{array}$ \\
\hline Police nationale & 23 \\
\hline Gendarmerie nationale & 1 \\
\hline Administration pénitentiaire & 17 \\
\hline Total général & 41 \\
\hline
\end{tabular}

Tableau I. Coût total des gardes et escortes de détenus hospitalisés en 2006 (d'après [5]).

la possibilité de s'affranchir de ces contraintes logistiques. Ceci est avantageux pour les établissements de détention, mais aussi pour les établissements de santé, avec des patients qui présentent parfois des difficultés de déplacement (personnes âgées dépendantes, handicap). Dans un autre contexte, notamment en gériatrie, où la santé des patients est déjà altérée, le dépistage et la prise en charge initiale de problèmes de santé bucco-dentaire s'inscrit dans une démarche de traitement global, indispensable au maintien de la qualité de vie des patients. Par cette approche, il n'est plus nécessaire de planifier le déplacement des patients et de mettre en place la logistique appropriée (ambulance, accompagnants).

\section{Organisation hiérarchisée des prises en charge}

À l'issue de l'expertise, un compte rendu détaillé dans lequel un niveau de priorité de soins est évalué, est réalisé par le service de médecine bucco-dentaire de l'hôpital Henri Mondor. Cette hiérarchisation permet un classement des priorités des patients qui nécessitent des soins urgents afin qu'ils soient repérés et puissent effectivement bénéficier des soins appropriés. Ce système fonctionne de pair avec les soignants de l'unité sanitaire de l'établissement pénitentiaire qui utilisent ces comptes-rendus afin de faciliter les séquences de prise en charge des patients.

\section{Confort pour le patient}

La téléexpertise améliore le confort du patient. Ceci n'est pas lié à l'absence du chirurgien-dentiste (vis-àvis duquel il peut ressentir une appréhension), celui-ci intervenant dans un second temps afin de réaliser les soins nécessaires, mais au fait que l'utilisation de la caméra intra-buccale n'est pas invasive et permet de le familiariser avec les soins. II s'agit donc d'une première étape qui facilite la coopération future du patient avec le praticien. Cet aspect est d'autant plus important pour les patients craintifs (ou en situation de handicap empêchant la communication) qui se verront proposer 


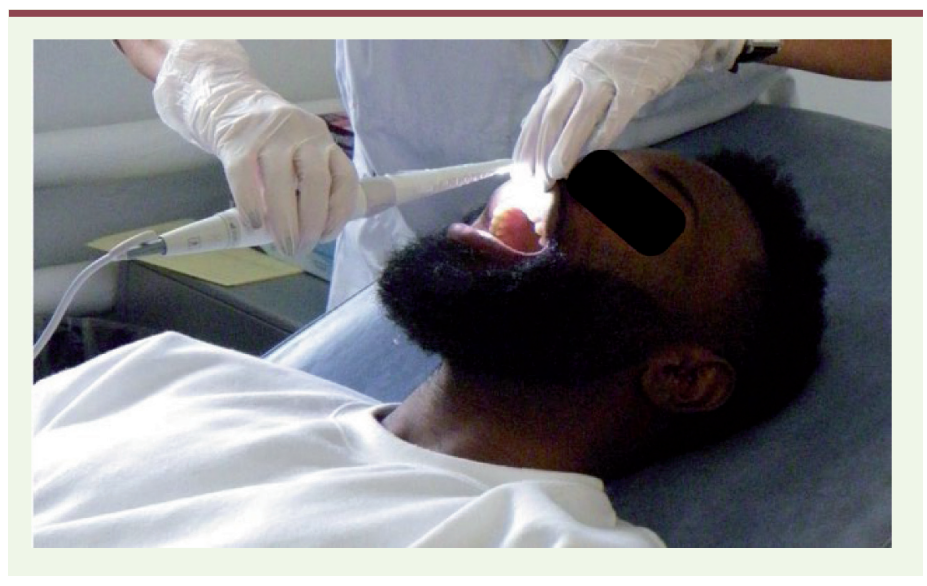

Figure 2. Acquisition d'images à l'aide de la caméra intra-orale.

une solution simple et efficace de dépistage. Notons également que l'absence de déplacement améliore le confort du patient.

\section{Absence de contraintes d'horaires}

La téléexpertise permet l'analyse différée des fichiers vidéo qui ont été enregistrés: ils sont stockés sur une plateforme commune entre les établissements dans l'attente d'être examinés. Ceci permet ainsi aux deux établissements de communiquer sans contraintes d'horaires et de disponibilité.

\section{Analyse et diagnostic}

Dans le cadre de leur mission de santé publique, les chirurgiensdentistes de l'hôpital Henri Mondor participent à la téléexpertise en analysant les vidéos reçues et en rédigeant les comptes rendus. Cette expérience leur permet de développer progressivement leurs facultés d'analyse (en ce qui concerne les lésions carieuses notamment) et de s'entraîner au dépistage par l'examen des nombreuses vidéos qui leur sont adressées. Ils sont ainsi amenés à poser un diagnostic en fonction des différents éléments observés, ce qui les entraîne également à la démarche diagnostique.

\section{Organisation de Télédent}

La téléexpertise s'appuie sur un dispositif simple dans lequel un personnel de santé, responsable de la prise en charge du patient, collecte des données auprès du patient à l'aide d'une caméra et d'un logiciel. Ces informations sont ensuite envoyées par l'intermédiaire d'une plateforme sécurisée à un chirurgien-dentiste qui les analyse et adresse un compte-rendu [5] $(\rightarrow)$. L'équipe de Télédent a procédé à une formation

$(\rightarrow)$ Voir le Repères de M. Allouche et al., $\mathrm{m} / \mathrm{s}$ $n^{\circ} 12$, décembre 2017, page 1105 initiale des personnels médicaux et paramédicaux de l'équipe requérante de l'unité sanitaire de l'établissement pénitentiaire de Fresnes. Au cours de cette formation, le matériel et la caméra intra-orale avec son logiciel associé ont été présentés. Une mise en pratique a été effectuée afin de préciser les éléments anatomiques bucco-dentaires qu'il était nécessaire d'enregistrer.
Après cette formation, Télédent a pu être mis en application. La séance débute par la réalisation, au sein de l'unité sanitaire de l'établissement pénitentiaire, de l'acquisition des images vidéo via la caméra intra-orale (Figure 2) [5]. Cette caméra est adaptée à tout type de bouche et s'utilise avec des étuis hygiéniques à usage unique. Les images recueillies sont ensuite transmises à l'équipe de l'hôpital Henri Mondor, via la plateforme ORTIF (Outil régional de télémédecine d'llle-de-France). Cette plateforme est un logiciel de télémédecine qui permet les activités de téléconsultation et de téléexpertise et les échanges d'information entre les différentes équipes, de façon organisée et sécurisée.

Chaque télédossier transmis aux chirurgiens-dentistes du service de médecine bucco-dentaire de l'hôpital comporte les informations anonymisées du patient. II comprend quatre vidéos présentant chacune des secteurs de sa cavité buccale et un questionnaire médical ciblé dans lequel figurent l'anamnèse, la symptomatologie, la présence ou non d'un trismus (ou contraction constante et involontaire des muscles de la mâchoire), la consommation de toxiques, etc. (Figure 3).

La visualisation des vidéos permet une analyse détaillée dent par dent. Le compte rendu précise, par exemple, les dents absentes ou l'état des racines, les lésions carieuses, les restaurations inadaptées, la présence de tartre, mais aussi la nécessité de réaliser des examens complémentaires, comme une image radiographie panoramique dentaire. Le niveau de priorité d'accès aux soins est finalement évalué à l'aide d'un codage allant de 1 à 4 (Tableau II).

\section{Limites et difficultés rencontrées}

L'acquisition des fichiers vidéo à partir de la caméra est relativement simple mais elle nécessite toutefois un apprentissage afin d'obtenir des vidéos de qualité permettant une analyse détaillée. L'une des difficultés principales a donc consisté, pour le personnel chargé de réaliser ces vidéos, en la manipulation de la caméra dans la cavité buccale, en enregistrant correctement les surfaces dentaires. L'analyse des fichiers par les chirurgiens-dentistes requiert également un certain entraînement pour la lecture des vidéos. L'analyse des tissus mous (langue, muqueuses) et le dépistage d'une éventuelle lésion muqueuse nécessite une expérience particulière à acquérir.

Pour l'équipe de Fresnes, l'introduction de la caméra dans la cavité buccale des patients représentait une difficulté, les intervenants n'étant pas habitués à une telle manipulation auprès du patient. Pour les patients, se voir proposer dès leur incarcération un examen buccal a également pu constituer une difficulté. L'appren- 


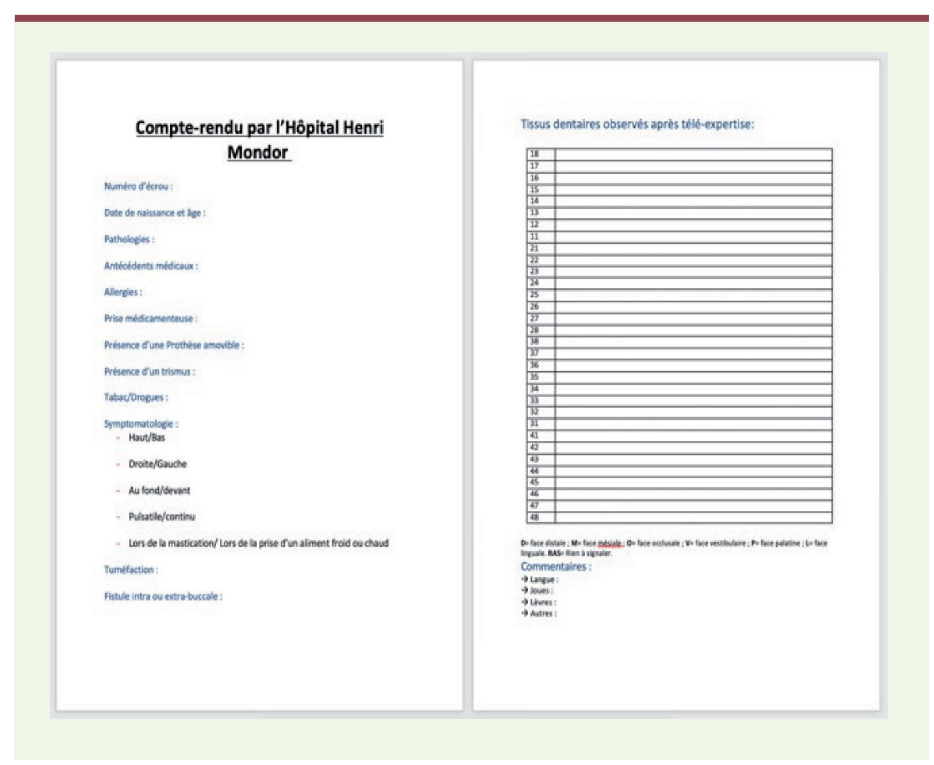

Figure 3. Fiche-modèle du compte rendu.

tissage progressif de la manipulation du dispositif et l'accélération des examens ont toutefois rapidement levé ces problèmes.

\section{Perspectives}

Le projet Télédent a vocation à être développé et étendu à d'autres établissements. Initialement limité au centre pénitentiaire de Fresnes, il devrait notamment être très utile dans les établissements médicaux comme les établissements d'hébergement pour personnes âgées dépendantes (EHPAD) et les institutions recevant les personnes souffrant d'un handicap mental ou moteur.

Les seules conditions à la mise en place de ce système sont la disponibilité du personnel et leur formation à la manipulation des vidéos ainsi qu'à l'utilisation de l'interface informatique pour la transmission des données. L'extension de la télémédecine bucco-dentaire à des établissements notamment scolaires pourrait aussi être une éventualité intéressante à considérer dans une optique de dépistage et de prévention. La caméra intra-orale rend possible l'observation et l'analyse relativement fiables des surfaces dentaires, permettant le dépistage des lésions carieuses, des lésions d'usures et autorisant l'examen des restaurations prothétiques en place. II n'est cependant pas encore possible d'examiner avec une grande précision les muqueuses et, de fait, les potentielles lésions les touchant. Dans une optique de recherche et de développement, il est donc nécessaire d'incorporer dans un avenir proche une fonction d'analyse plus précise des tissus mous afin d'éviter au patient une possible perte de chance quant à la prise en charge d'une lésion suspecte.

\section{Conclusion}

La téléexpertise en milieu pénitentiaire permet un dépistage des pathologies dentaires, avec les mêmes exigences de qualité et de sécurité qu'un dépistage en présentiel. Elle permet une amélioration

1 Pas de nécessité de soins apparente
2 Nécessité de soins, mais non urgente
3 Soins à réaliser au plus vite
4 Urgence

Tableau II. Niveau d'urgence de réalisation de soin.

de l'efficience et de l'organisation des soins dentaires dans une maison d'arrêt. Le taux de refus du bilan par téléexpertise est très faible par rapport au taux habituel de refus des consultations dentaires $(4,1 \% /$ $23 \%)$. Cela pourrait résulter des explications apportées par l'équipe au patient et à l'absence de douleurs, et également de la rapidité des rendez-vous donnés et de la localisation sur site dans la division où le patient est détenu. Lorsque les vidéos leur sont présentées, les patients prennent aussi conscience de la dégradation de leur état bucco-dentaire et des actions de prévention peuvent être réalisées.

Ce modèle de travail avec l'unité sanitaire de Fresnes pourra être appliqué et adapté à d'autres structures, où l'intérêt d'avoir des consultations et des suivis réguliers sans besoin de déplacement sont importants, notamment les établissements de gériatrie.

Télédent n'a pas pour objectif de remplacer les actes bucco-dentaires en présentiel. L'examen d'une vidéo ne présente pas la même précision qu'un examen clinique réalisé avec l'œil du praticien, directement en bouche, et ne remplace pas les tests et les examens complémentaires d'une consultation classique. Cependant Télédent constitue une réponse aux difficultés de l'offre de soins dans le milieu pénitentiaire. II s'agit maintenant de développer ce modèle par la formation du personnel, la motivation des personnes détenues, et la valorisation financière des actes réalisés. $\diamond$

\section{SUMMARY}

Télédent, an oral tele-expertise experience in a penitentiary environment

The oral health has an important role in the good general health state. Some populations, including those in detention, have not only increased needs, but also limited access to dental care. Télédent is an oral tele-expertise activity that facilitates the screening and prevention of oral disorders, performing as a complementary health tool for screening. With an intra-oral camera, oral images of the prisoners are recorded and then analyzed by a team from the Oral Medicine Department of the Hospital Henri Mondor, giving the UCSA of Fresnes prison a detailed dental expertise. As a result, Télédent enables a cooperation 
to improve the screening and oral care of prison inmates at the Fresnes Prison, decreasing costs and increasing the security. $\diamond$

\section{LIENS D'INTÉRÊT}

Les auteurs déclarent n'avoir aucun lien d'intérêt concernant les données publiées dans cet article.

\section{RÉFÉRENCES}

1. Santé H-HAd. Stratégies de prévention de la carie dentaire. Recommandations en Santé Publique 2010. https://www.has-sante.fr/upload/docs/application/pdf/2010-10/corriges_synthese_ carie_dentaire_version_postcollege-10sept2010.pdf

2. Oberlé $D$. Les soins et la prévention bucco-dentaire dans les établissements pénitenciaires. Étude et Recherche Odontologie - Odontefr 2016. https://odonte.com/index.php/2016/01/22/soinsdentaires-dans-les-etablissements-penitentiaires/.
3. Fardel É. Promotion de la santé bucco-dentaire au centre de détention de Mauzac. Thèse d'exercice en chirurgie dentaire. Université de Bordeaux 2017. https://dumas.ccsd.cnrs.fr/dumas-01511614.

4. Ollivier $Y$, Blot $Y$, Debernardy $B$, et al. Transfert à l'administration pénitentiaire de la mission de garde et d'escorte des détenus hospitalisés. Ministère de l'intérieur 2007 ; Rapport de l'Inspection Générale de l'Administration (en ligne) https://www.interieur.gouv.fr/Publications/ Rapports-de-I-IGA/Securite/Transfert-a-I-administration-penitentiairede-la-mission-de-garde-et-d-escorte-des-detenus-hospitalises [consulté le 21/05/2019: 1-130.

5. Allouche $M$, Herve $C$, Pirnay $P$. Le nécessaire questionnement éthique autour de la relation de soin en télémédecine bucco-dentaire. Med Sci (Paris) 2017 ; 33 : 1105-9.
TIRÉS À PART

A. Novais

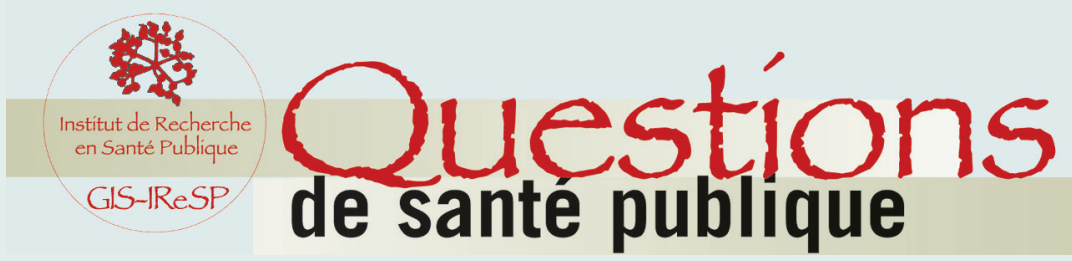

\section{Un nouveau bulletin}

pour une meilleure

visibilité des résultats

de la recherche

en santé publique

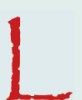

es résultats de la recherche en santé publique souffrent en France d'un réel manque de visibilité. Ceci concerne aussi bien le monde académique (hors santé publique) que le grand public et les décideurs. Pour pallier ce déficit, I'IReSP a créé un bulletin à large diffusion intitulé "Questions de santé publique ", largement inspiré du bulletin mensuel d'information de I'INED " Populations et sociétés ". L'objectif éditorial est de porter à la connaissance d'un large public (enseignants, étudiants, journalistes, décideurs, milieux de la recherche, associations, public concerné) les informations les plus récentes concernant des questions importantes de santé publique, rédigées de façon facilement lisible et compréhensible pour des non spécialistes, en garantissant que les informations publiées sont validées scientifiquement. La publication concerne des faits et non des positions. Audelà de la présentation de résultats, les qualités pédagogiques de Questions de santé publique permettent au lecteur de mieux comprendre comment sont formulées et abordées les questions de santé publique et quelles sont les limites de ces études.

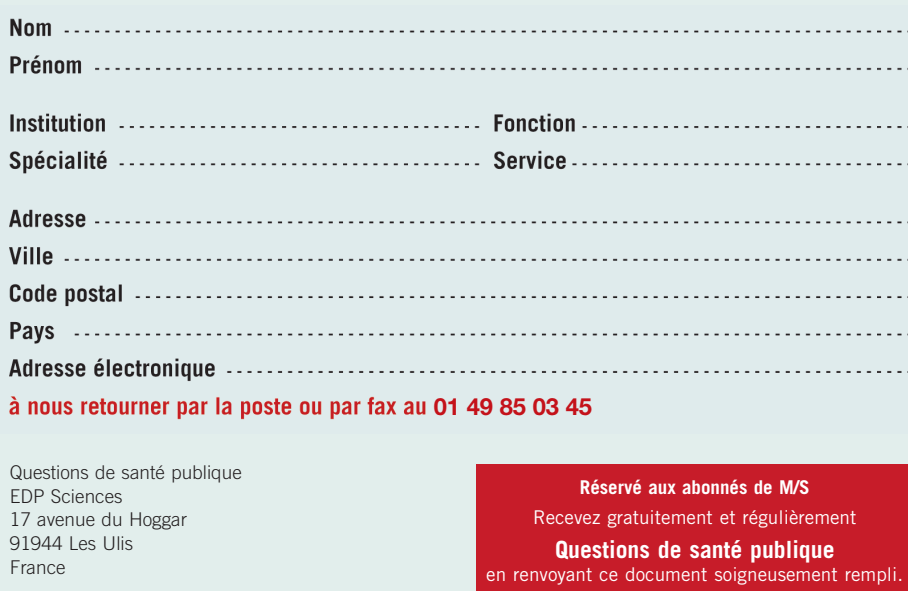

Questions de santé publique est une publication de l'Institut de Recherche en Santé Publique I Directeur de la publication : Corinne Alberti. I Rédactrice en chef : Kodja Yetongnon. I Comité de relecture : Lorraine Cousin, Jean-Marie Gagliolo, Coline Terroba. I Réalisation : EDP Sciences. 